\title{
Motivação: Ferramenta de Trabalho do Enfermeiro na Prática da Educação em Saúde na Atenção Básica
}

\author{
Motivation: A Work Tool for the Nurse in the Practice of \\ Health Education in Primary Care
}

\author{
JULIANA MARQUES WEYKAMP ${ }^{1}$ \\ DIANA CECAGNO ${ }^{2}$ \\ PATRICIA PAZ HERMEL ${ }^{3}$ \\ FERNANDO DALMASO TOLFO ${ }^{3}$ \\ HEDI CRECENCIA HECKLER DE SIQUEIRA ${ }^{4}$
}

\section{RESUMO}

Introdução: A educação em saúde direciona o processo de trabalho na construção de novas práticas profissionais, a partir de diferentes formas de utilização do conhecimento. Dessa forma, entende-se que a prática educativa pode ser considerada como uma importante estratégia no intuito de oportunizar a qualificação do cuidado individual e coletivo, uma vez que ela é percebida como um processo de trocas de saberes e experiências entre os profissionais, os gestores e os usuários dos serviços e instituições de saúde. Objetivo: Conhecer a percepção do enfermeiro acerca da motivação como ferramenta de trabalho na prática da educação em saúde, no âmbito da atenção básica. Material e métodos: Estudo descritivo, exploratório com abordagem qualitativa, realizado com 11 enfermeiros que trabalham em unidades básicas de saúde num município do Estado do Rio Grande do Sul. Os dados foram coletados por meio de entrevistas semiestruturadas e analisados seguindo a Análise Temática. Resultados: Os resultados possibilitam inferir que a educação em saúde é uma atividade realizada com o propósito de fornecer subsídios para o autocuidado, fortalecer a autonomia e a conquista da cidadania do usuário. Conclusão: A motivação é percebida como instrumento de trabalho na prática da educação em saúde, podendo ser uma estratégia para dar maior visibilidade ao trabalho do enfermeiro, fortificálo na sua autonomia profissional uma vez que suas funções, legalmente exercidas, envolvem atividades educativas, direcionadas às questões de saúde da população.

\section{DESCRITORES}

Enfermagem. Motivação. Educação em Saúde.

\begin{abstract}
Introduction: Health education guides the work process towards the development of new professional practices by using the knowledge through different approaches. Thus, it is understood that educational practice can be considered as an important strategy to create opportunities for qualification of individual and collective care. It is perceived as a process of knowledge and experience exchange among professionals, managers and users in healthcare services and institutions. Objective: To know the perception of nurses about motivation as a tool in the practice of health education in primary care. Material and methods: This was a descriptive, exploratory study with a qualitative approach including 11 nurses working in primary health care units in the State of Rio Grande do Sul, Brazil. Data were collected through semi-structured interviews and analyzed according to thematic analysis. Results: The results allow inferring that health education is an activity carried out to support self-care, strengthen autonomy and reaffirm the user's citizenship. Conclusion: Motivation is perceived as a working tool in the practice of health education. It may be a strategy to give greater visibility to the work of nurses, as well as to encourage their professional autonomy since their duties include educational activities focused on community health issues.
\end{abstract}

\section{DESCRIPTORS}

Nursing. Motivation. Health Education.

1 Mestranda do Programa de Pós-Graduação em Enfermagem (PPGENF/FURG/). Pelotas/ RS, Brasil.

2 Docente do Departamento de Enfermagem da Universidade Federal de Pelotas (FEN/UFPEL). Doutoranda do Programa de Pós-Graduação em Enfermagem (PPGENF-FURG/RS). Pelotas/RS, Brasil.

3 Enfermeira da Fundação Estatal de Atenção especializada em saúde. Curitiba/PR, Brasil.

4 Docente permanente do Programa de Pós-Graduação em Enfermagem (PPGENF/FURG)Pelotas/RS, Brasil 
$\mathrm{A}$ mudança de paradigma acerca da concepção de saúde e educação, que vem acontecendo nas últimas décadas, oportunizou a modificação na compreensão do entendimento, aplicabilidade e da nomenclatura de "educação para a saúde", em que o indivíduo tinha que aprender para cuidar de si e evitar doenças, para "educação em saúde", na qual o indivíduo troca experiências e saberes entre si e com os profissionais para cuidar de sua saúde. No entanto, autores destacam que para haver uma ruptura completa desse paradigma, é necessário que haja mudança de comportamentos e atitudes em relação à saúde individual e coletiva, tanto dos profissionais quanto dos indivíduos, fazendo com que as práticas de educação, especialmente, da promoção em saúde, sejam efetivamente utilizadas no cotidiano de vida e de trabalho dos envolvidos ${ }^{1}$.

Entende-se que a mudança consciente ocorre com a participação efetiva do usuário/família nos meios de produção de conhecimentos e de habilidades para atuar frente às ações na promoção da saúde. Esta forma de participação é compreendida como um método consciente e crítico, em que a mudança de comportamento que visa o cuidado em saúde acontece por meio da aprendizagem em saúde, que ocorre pelo entendimento das condições dos serviços de saúde oferecidos e a forma como os profissionais, pertencentes a estes serviços de saúde, atuam junto ao usuário ${ }^{2}$. Entretanto, torna-se necessário considerar, nessa conscientização crítica, o meio ambiente em que o ser humano vive, trabalha e se desenvolve porque esse exerce interação e influência em sua saúde ${ }^{3}$.

$\mathrm{O}$ enfermeiro, como integrante da equipe de saúde, necessita empoderar-se de estratégias que possibilitem atender o indivíduo, a família e a comunidade de forma integral e contínua, desenvolvendo ações de promoção, proteção e manutenção da saúde. Neste sentido, entende-se que a prática educativa pode ser considerada como uma importante estratégia no intuito de oportunizar a qualificação do cuidado individual e coletivo, uma vez que ela é percebida como um processo de trocas de saberes e experiências entre os profissionais, os gestores e os usuários dos serviços e instituições de saúde $^{4}$. Os autores corroboram quando referem que ensinar a comunidade pode ser uma estratégia de cuidado, sendo muito explorada pelos enfermeiros ${ }^{5}$. De igual forma, pode tornar-se uma importante ferramenta para o cotidiano de trabalho do enfermeiro, a fim de motivar a modificação de comportamentos e atitudes que possibilitem a melhoria da qualidade de vida, a promoção da saúde e da autonomia dos sujeitos envolvidos, tornando-os ativos, críticos e transformadores de um existir saudável e com qualidade.

A educação em saúde direciona o processo de trabalho na construção de novas práticas profissionais a partir de diferentes formas de utilização do conhecimento. Neste interim, o cotidiano de trabalho da enfermagem induz a constantes reflexões decorrentes das mudanças políticas, econômicas, educacionais e sociais que atingem a estruturação e organização das instituições e serviços de saúde ${ }^{6}$.

Ao perceber a educação em saúde como uma ferramenta de trabalho essencial na prática cotidiana, o enfermeiro pode expandir, de forma significativa, enquanto educador, a sua parcela de contribuição frente ao cuidado aos usuários, famílias e comunidade, desenvolvendo, com a competência técnica, cientifica e legal que lhe é garantido por lei, sua função de educador, de promotor da saúde. Ainda, essas práticas tornam-se uma importante estratégia para os enfermeiros que querem fazer diferença na sua trajetória profissional, independente do local em que desenvolvem suas práticas cotidianas. Isto por que a educação em saúde surge como prática social no âmbito das ações desenvolvidas pelos profissionais enfermeiros. Entretanto, ela necessita estar à disposição do usuário, tornando-o autônomo e capaz de se reajustar às necessidades que se apresentem ${ }^{7}$. As práticas educativas, tanto podem ser formais como informais e são possíveis de serem desenvolvidas em qualquer espaço onde dois ou mais indivíduos estiverem reunidos, desde que atendam os imperativos das pessoas envolvidas no contexto ${ }^{8}$.

Neste ínterim, enfatiza-se a necessidade de que estas atividades sejam percebidas como uma importante estratégia capaz de motivar/estimular mudanças de comportamentos e atitudes a fim de promover hábitos saudáveis de vida, com o intuito da manutenção da saúde individual e coletiva, tornando os usuários cada vez mais comprometidos com a sua qualidade de vida e sua condição de cidadão. Por entender a relevância do tema, objetivou-se conhecer a percepção do enfermeiro acerca da motivação como ferramenta de trabalho na prática da educação em saúde, no âmbito da atenção básica.

\section{MATERIAL E MÉTODOS}

Trata-se de um estudo descritivo e exploratório, com abordagem qualitativa. Participaram do estudo 11 enfermeiras, que trabalham em Unidades Básicas de Saúde(UBS) num município ao Sul do Estado do Rio Grande de Sul. Foram respeitadas as recomendações da Resolução 466/12 do Conselho Nacional de Saúde, que regulamenta investigações envolvendo seres humanos. A pesquisa foi submetida ao Comitê de Ética em Pesquisa com Seres Humanos do Hospital Santa Casa de Misericórdia de Pelotas, aprovado sob o protocolo ${ }^{\circ} 180 / 2012$. 
No intuito de atender ao objetivo proposto, optou-se pela realização de entrevistas semiestruturadas que foram realizadas em encontros individuais, no local de trabalho de cada participante. A fim de preservar o anonimato, os participantes foram identificados com a letra E, seguida de um numeral que sinaliza a ordem da realização das entrevistas: E1, E2 ....E11. As informações coletadas durante a entrevista foram gravadas e transcritas, pela autora da pesquisa, logo após o término da mesma, a fím de preservar a veracidade dos dados.

A análise dos dados deu-se por meio da análise temática para tratamento dos dados, que "consiste em descobrir os núcleos do sentido que compõe uma comunicação, cuja presença ou frequência signifique alguma coisa para o objeto analítico visado" 9 .

\section{RESULTADOS}

A partir da análise dos dados buscou-se agrupar as unidades de registro que evidenciaram a motivação como ferramenta de trabalho do enfermeiro nas ações educativas em saúde.

Buscou-se, primeiramente, o entendimento dos participantes acerca do conceito e aplicabilidade da educação em saúde no seu cotidiano. A seguir questionou-se a respeito sobre a sua percepção em relação à motivação como instrumento de trabalho na prática da educação em saúde.

Quanto ao entendimento do conceito e aplicabilidade da educação em saúde, no ambiente da atenção básica, os participantes se expressaram conforme as falas a seguir:

Educação em saúde é uma forma de ensinamento direcionada para os usuários a fim de promover o autocuidado [...] serve para prevenir doenças como também ajudar no tratamento de algo já estabelecido (E1),

[...] é poder educar a população que tu assiste em relação à prevenção, orientar quanto à alimentação, quanto a uma vida melhor, em todo, assim tu poder observar as necessidades daquela família e dentro disso começar o teu trabalho de educação(E3),

Acho que é um conjunto de saber e práticas para a prevenção de doença(E4).

A partir das falas das enfermeiras entrevistadas pode-se inferir que a educação em saúde é uma atividade com o propósito de prover conhecimentos com a finalidade de orientar os usuários dos serviços de saúde, em relação às suas dúvidas e fornecer subsídios para o autocuidado, fortalecer a sua autonomia visando a conquista da sua cidadania. Embora esteja enfatizado a questão do orientar ou ensinar por meio da socialização do conhecimento, fato embasado nas características da formação profissional do enfermeiro, pode-se observar que o entendimento atribuído pelas participantes corrobora que a educação em saúde pode propiciar a interação com o individuo e/ou coletividade, incluindo o contexto, as necessidades e escolhas, bem como valorizando a autonomia para assumir sua própria saúde, a partir da concepção do conceito saúdecuidado-doença ${ }^{10}$.

Entretanto, existe um significado diferente entre os conceitos, enquanto na prática de ensinar não há troca de informações, os indivíduos tornam-se meros espectadores e receptores de informações sem participar da busca de soluções para seus problemas, a prática de educar remete a um processo dialógico, ou seja, um intercâmbio de idéias onde há respeito e valorização do conhecimento e participação e autonomia do sujeito nas ações relativas ao seu bem-estar ${ }^{11}$. Entende-se que educar para melhorar a qualidade de vida implica em mudança de comportamento e atitudes tanto do profissional que educa, como também do usuário que recebe as orientações, ambos sujeitos do processo, conforme está salientado que ninguém educa ninguém, que ninguém educa a si mesmo, e que os homens se educam entre si mediados pelo universo ${ }^{12}$. Corroborando com o autor, a educação ambiental e a educação em saúde ensinam a pensar sobre como as mais diversas ações individuais podem refletir nas condições de vida e saúde de uma comunidade ${ }^{13}$. Portanto, é necessário ensinar a pensar na relação complexa que existe entre homem, saúde e ambiente.

Assim, o enfermeiro ao utilizar, na atenção básica, a motivação como ferramenta de trabalho na prática da educação em saúde, na atenção básica, busca incentivar e estimular o usuário a tomar-se parte na educação em saúde, deixando-o livre e autônomo no seu processo de viver saudável. A educação em saúde, utilizada, como ferramenta de motivação, foi percebida pelas entrevistadas como:

A educação em saúde tem o objetivo de desenvolver a autonomia do usuário pra que ele desenvolva as coisas sozinho, desenvolva o autocuidado (E2),

Sim [...] agora mesmo tava atendendo um paciente com problema renal, fiz um desenho do rim e expliquei pra ele toda função do rim, a importância que órgão tem no organismo, porque que ele deve cuidar e tomar bastante liquido (E10),

Sim, nos atendimentos do dia-a-dia, dando orientações, nas visitas domiciliares. A gente faz educação em saúde todo tempo aqui dentro (E5). 
Entretanto, algumas das enfermeiras entrevistadas utilizam o termo ensinar e orientar como se tivessem o mesmo sentido e definem a Educação em saúde e direcionam a atividade para prevenir doenças e auxiliar em tratamentos.

Em relação às práticas capazes de possibilitar o autocuidado, essas devem oportunizar aos indivíduos desempenhá-las de forma consciente e voluntária, em seu benefício e com o propósito de manter o seu bemestar. Se assim são percebidas, podem auxiliar para que o usuário se posicione de forma crítica, refletindo sobre sua condição de sujeito comprometido com a sua realidade $^{14}$. Essa atitude torna-o co-responsável pelas ações de sua saúde, e participante do seu plano de cuidado.

O envolvimento dos usuários na implementação do seu plano de cuidado é fundamental, e cabe à equipe motivá-los a desenvolver suas capacidades e explorar seus potenciais em função de sua idade, estilo de vida, condições e exigências cotidianas, a fim de melhorar sua qualidade de vida ${ }^{15}$. A Teoria do Autocuidado tem como base o pressuposto de que todos os seres humanos têm potencial para desenvolver habilidades intelectuais e práticas para o autocuidado $^{16}$.

Neste ínterim, a educação para o autocuidado traz à tona o preconizado na teoria do autocuidado, que tem como proposta possibilitar aos indivíduos, família e comunidade tomarem iniciativas e assumirem responsabilidades no desenvolvimento de seu próprio caminho em direção à melhoria da qualidade de vida, saúde e bem-estar ${ }^{17}$. Na prática do autocuidado, cinco atribuições devem ser seguidas pelo profissional educador: manutenção do autocuidado; prevenção do não cuidado; autodiagnóstico; automedicação e autotratamento e participação nos serviços de atenção à saúde e aos agravos à saúde.

A educação em saúde foi mencionada como uma prática inserida na consulta de enfermagem, utilizada na atenção básica. Esta ação está respaldada na Lei do Exercício Profissional n. ${ }^{\circ} 7.498$, de 25 de junho de 1986, e o Decreto 94.406/87 que legitimaram a Consulta de Enfermagem como uma atividade privativa do enfermeiro com o objetivo de instituir-lhe a autonomia nas ações de promoção, proteção, recuperação ou reabilitação da saúde ${ }^{18}$.

Também é possível apreender que as entrevistadas consideram as atividades desenvolvidas em grupo nos programas de atenção à saúde do Ministério da Saúde, como sendo ações de educação em saúde:

[...] também nos grupos se orienta a importância da alimentação e exercícios regulares (E4),
Sim, nos grupos do hiperdia e das gestantes, durante um atendimento e outro da demanda aqui na unidade (E1).

Neste caso, é importante salientar que, embora haja consciência da importância das atividades de educação em saúde, a prática destas está diretamente relacionada às atividades desenvolvidas durante os grupos de gestantes e do HiperDia que é um programa governamental que cadastra e acompanha pacientes hipertensos e/ou diabéticos, captados e vinculados às Unidades Básicas de Saúde (UBS), e que geram informações para profissionais de saúde e gestores públicos ${ }^{19}$. Em contrapartida, a realização de encontros com os usuários a fim de praticar ações educativas não ocorre com frequência no cotidiano de trabalho do enfermeiro e equipes, constituindo-se, assim, em lacunas no desenvolvimento dos programas oferecidos pelo governo.

Em relação à atividade educativa do enfermeiro, estes precisam oferecer espaços de liberdade, incentivar o questionamento, a curiosidade, além de educar para o desenvolvimento do pensamento e da coragem de agir ${ }^{20}$. Desta forma, inserir a motivação na educação em saúde como um instrumento de trabalho, pode auxiliar a direcionar as ações do cotidiano do enfermeiro a fim de empoderá-lo por meio da utilização de novos formatos de planejamento, organização e avaliação do seu trabalho.

$O$ fato de alguns entrevistados se perceberem realizando atividades educativas junto aos grupos do HiperDia, com a entrega de medicação, contradiz o que a literatura aborda acerca do tema. $\mathrm{O}$ simples fato, de entregar medicação para o usuário hipertenso e/ou diabético satisfaz apenas um dos segmentos do todo que deveria ser realizado. Essas brechas constituem-se em vazios no desenvolvimento dos programas oferecidos pelo Ministério da Saúde à população.

Os dados levam a inferir que a concepção que cada enfermeiro tem acerca da educação em saúde, influencia no modo como estas práticas são desenvolvidas na UBS. E, em consequência, no tipo de vinculo mantido com os usuários. Neste contexto, percebe-se que o princípio para realizar as atividades de educação em saúde é conquistar a confiança do usuário, família e comunidade, construindo um vínculo favorável. A conquista do reconhecimento e da visibilidade do enfermeiro, muitas vezes, alicerçados pela aquisição de conhecimento e ferramentas favorecem o desenvolver do seu fazer, condicionam sentimentos de confiança frente aos demais ${ }^{21}$. O vínculo construído com os usuários que procuram os serviços e instituições de saúde fortalece as ações educativas e proporciona a sua maior adesão, bem como possibilita a formação de sujeitos autônomos frente a sua condição de saúde. 
Para que haja a formação de vínculos, a equipe de trabalho deve ser receptiva, comprometida e qualificada, uma vez que devem atuar tendo em mente as especificidades individuais e coletivas, pois o comprometimento com o trabalho estabelece uma relação de confiança e inspira motivação para a adesão ao cuidado proposto ${ }^{22}$.

A relação de confiança construída entre o enfermeiro e o usuário poderá ser gradativa, sendo necessário um investimento do profissional, utilizando a criatividade, capacidade de inovação, comprometimento e interação com o usuário. Essa maneira de ser e agir pode contribuir a fim de garantir a co-responsabilidade do usuário com os profissionais e produzir resultados positivos em benefício de sua saúde. Ao estabelecer uma relação de confiança é preciso percorrer um processo dinâmico no qual os enfermeiros e os clientes são atores aliados e em constante construção/reconstrução ${ }^{23}$.

A habilidade e a criatividade do enfermeiro, ao realizar ações de educação em saúde com os usuários, pode contribuir positivamente nos resultados das práticas implementadas com a população, fazendo com que estas ações sejam de fácil abordagem, melhor compreendidas, tendo assim, maior probabilidade de serem colocadas em prática.

Com o entendimento que a motivação pode ser considerada um instrumento importante no cotidiano de trabalho do enfermeiro, questionou-se os entrevistados acerca dos fatores responsáveis pela sua motivação. Por meio das falas a seguir é possível identificar fatores desencadeadores da motivação:

Eu me sinto motivada, porque eu gosto de atividades de educação em saúde [....] elas, para mim se sobressai em relação a qualquer outra atividade(E5),

Eu me sinto motivada por mim mesma, pelos outros não(E7),

$A$ vaidade pessoal de tu conheceres sobre o assunto e poder discorrer adequadamente sobre ele(E8), A importância do conhecimento no e para o cuidado do paciente (E10).

Os relatos identificam que os sujeitos se sentem motivados por fatores pessoais internos, que influenciam tanto por motivos profissionais como na realização pessoal. Os motivos mencionados induzem a pensar que é importante para os entrevistados que se sintam protagonistas de sua motivação. Isto vai ao encontro de que a motivação dos sujeitos se dá de maneira intrínseca, onde cada um demonstra impulsos motivacionais de formas e em períodos distintos, entendendo que estas forças afetam diretamente a forma de desenvolver as suas atividades e o viver de suas próprias vidas $^{24}$.

Neste ínterim, a motivação interna abarca situações de sentimentos dos indivíduos e devem ser desenvolvidas, pois é ela que adquire maior valor e alcança o melhor objetivo que é o da participação e envolvimento nas atividades planejadas e implementadas, gerando o comprometimento de cada profissional $^{7}$. As autoras também enfatizam que é necessário, além de incentivar, proporcionar condições adequadas para a sua concretização e isso pode ser possível por meio da inclusão dos profissionais no planejamento e implantação de estratégias desenvolvidas pelas instituições e serviços.

Corroborando com a ideia, no cotidiano prático do enfermeiro, é impar a necessidade de proporcionar estímulos que impulsionem a criatividade do sujeito, despertando, assim, suas potencialidades para o desencadear de transformações necessárias no ambiente em que se encontra ${ }^{26}$. Desta forma, as autoras salientam que respeitando o tempo e as particularidades de cada sujeito no reconhecimento de suas potencialidades e, satisfazendo suas necessidades de pertença ao grupo/ equipe, o sujeito aumenta sua capacidade de produzir, de forma crítica e reflexiva, um conhecimento que impulsiona mudanças na organização do trabalho, na forma de ser e agir e na melhoria da qualidade de vida.

Há várias possibilidades que podem ser utilizadas, pelo profissional enfermeiro, para a prática de ações educativas em saúde. Por conseguinte, é preciso que diferentes configurações, de educação em saúde, sejam exploradas e empreendidas durante o seu cotidiano de trabalho com o usuário, buscando desenvolver um ambiente de comunicação, diálogo, resolubilidade, responsabilização e autonomia. Estas características devem estar pautadas, na motivação, no diálogo, no desenvolvimento da espiritualidade, no estimulo à reflexão e no trabalho em grupo ${ }^{7}$. Segundo as autoras, a motivação associada às demais estratégias, torna-se fundamental, pois, uma vez motivado, o ser humano sente-se estimulado a aprender, a inovar, a criar e a buscar desenvolver-se como sujeito de sua história e melhorar sua condição de existir no planeta terra, visto atualmente como um mundo turbulento, globalizado e em constante transformação.

\section{CONCLUSÃO}

Diante dos resultados obtidos, infere-se que a motivação potencializa as ações de educação em saúde no intuito de estimular o autocuidado dos usuários dos serviços e instituições de saúde, possibilitando ampliar a sua autonomia e co-participação na promoção da saúde. Inserir a motivação como ferramenta de trabalho, 
na proposta de educação em saúde, potencializa as ações do cotidiano do enfermeiro a fim de empoderá-lo para a utilização de novas maneiras de planejamento, organização e avaliação do seu trabalho.

\section{REFERENCIAS}

1. Figueira AB, Amestoy SC, Cecagno D, Tristão FS, Lima L, Correa VA. Visão do enfermeiro frente à prática da educação em saúde no ambiente hospitalar. Rev Cogitare Enferm. 2013; 18(2):310-316.

2. Machado MFAS, Vieira NFC, Silva RM. Compreensão das mudanças comportamentais do usuário no Programa Saúde da Família por meio da participação habilitadora. Ciência \& Saúde Coletiva. 2010; 15(4):2133-2143.

3. Santos MC, Siqueira HCH, Silva JRS. Saúde coletiva na perspectiva ecossistêmica: uma possibilidade de ações do enfermeiro. Rev Gaúcha Enferm. 2009; 30(4):750754.

4. Alves VS. Um modelo de educação em saúde para o Programa Saúde da Família: pela integralidade da atenção e reorientação do modelo assistencial. Interface Comunic., Saúde, Educ. 2005; 9(16):39-52.

5. Jahn AC, Guzzo PCC, Silva MC, Silva EBG, Lima EJ, Lima SBS. Educação popular em saúde: metodologia potencializadora das ações do enfermeiro. Rev Enferm, UFSM. 2012; 2(3):547-552.

6. Azzolin GMC, Mancio MLRM. Ação educativa como instrumento de trabalho do enfermeiro: revisão bibliográfica [Monografia] Residência em Enfermagem em Clínica Cirúrgica do Hospital e Maternidade Celso Pierrô; 2009.

7. Cecagno D, Siqueira $\mathrm{HCH}$. Educação continuada: um novo modelo de ensino na enfermagem - incubadora de aprendizagem. Pelotas: Ed. Universitária PREC / UFPEL; 2006.

8. Pacheco JA. Processos e práticas de educação e formação. Para uma análise da realidade portuguesa em contextos de globalização. Rev. Port. de Educação. 2009; 22(1): 105-43.

9. Minayo MCS. O desafio do conhecimento: pesquisa qualitativa em saúde. $6^{\mathrm{a}}$ ed. São Paulo (SP): Abrasco 2010.

10. Santos RV, Penna CMM. A educação em saúde como estratégia para o cuidado à gestante, puérpera e ao recém-nascido. Texto Contexto Enferm. 2009; 18(4): 652660.

11. Alvim NAT, Ferreira M de A. Perspectiva problematizadora da educação popular em saúde e a enfermagem. Texto e Contexto Enfermagem. 2007; 16(2):315-319.

12. FREIRE, Paulo. Pedagogia do oprimido. $30^{\mathrm{a}}$ ed. Rio de Janeiro (RJ): Paz e Terra; 1987.

13. Pereira CAR, Fernandes ALT, Silva IAV. A educação ambiental e em saúde no acolhimento e assistência infantil. In: II SEAT - Simpósio de Educação Ambiental e Transdisciplinaridade UFG/IESA/NUPEAT, 2011, Goiânia, Brasil.

14. Bub MBC, Medrano C, Silva CDda, Wink S, Liss PE, Santos EKAdos. A noção e cuidado de si mesmo e o conceito de autocuidado na enfermagem. Texto Contexto Enferm. 2006; 15 (Esp):152-157.

15. Silva TR, Feldmam C, Lima MHA, Nobre MRC, Domingues RZL. Controle de Diabetes mellitus e hipertensão arterial com grupos de intervenção educacional e terapêutica em sequimento ambulatorial de uma Unidade Básica de Saúde [Internet]. 02 out 2009. [Acesso em: 14 jun 2014]. Disponível em: http:// www. portaleducacao.com.br/medicina/artigos/5502/ controle-de-diabetesmellitus-e-hipertensao-arterial-comgrupos-de-intervencao.
16. Luz ALA, Silva GRF, Luz MHB. Teoria de Dorothea Orem: uma análise da sua aplicabilidade na assistência a pacientes estomizados. Rev Enferm UFPI. 2013; 2(1):6770.

17. Orem, DE. Self-care theory in nursing: Selected papers of Dorothea Orem. K. M. Renpenning, \& S. G. Taylor (Eds.). Springer Publishing Company. 2003.

18. Lei do exercício profissional $n^{\circ} 7.498$ e o Decreto $94.406 /$ 87 DECRETO N 94.406/87 (BR) [internet]. Dispõe sobre o exercício da Enfermagem, e dá outras providências [acesso em: 13 out 2014]. Disponível em:

http://www.coren-ro.org.br/decreto-n-9440687-dispoesobre-o-exercicio-da-enfermagem-e-da-outrasprovidencias 767.html

19. HIPERDIA [Internet]. Brasília: Ministério da Saúde (BR) [Acesso em 06 jan 2015]. Disponível em: http:// hiperdia.datasus.gov.br/.

20. Waldow VR. Momento de cuidar: momento de refle Momento de cuidar: momento de reflexão na ação. Rev Bras Enferm. 2009; 62(1): 140-145.

21. Avila LI, Silveira RS, Lunardi VL, Fernandes GFM, Mancia JR, Silveira JT. Implicações da visibilidade da enfermagem no exercício profissional. Rev Gaúcha Enferm 2013; 34(3):102-109.

22. Martins AR, Pereira DB, Thofehrn MB, Cecagno D, Hisse CN, Quadros LCM. de. O resgate das relações de trabalho em uma equipe de Saúde da Família do município de Pelotas. In: Thofehrn MB. Práticas de gestão e gerenciamento no processo de trabalho em saúde. Pelotas: Editora Universitária / UFPEL; 2009. 240p.

23. Lourenço C, Pinto A, Pereira C, Fonseca C, Nunes I, Almeida MP, Mendes O, Tolleti G, Lopes M, Gândara, M.Confiança versus Desconfiança na Relação de Cuidar: Confiança Enfermeiro-Cliente, um Conceito em Construção no CHLN-HPV. Pensar Enfermagem. 2011; 15(2): 03-13.

24. Volpato M, Cimbalista S. O processo de motivação como incentivo à inovação nas organizações. Rev. FAE. 2002; 5(3):75-86.

25. Siqueira $\mathrm{HCH}$, ERDMANN AL. Construtivismo como método de pesquisa: possibilidade de Geração de conhecimentos. R Enferm UERJ. 2007; 15(2):291-297.
Correspondência
Juliana Marques Weykamp
Endereço: Rua Visconde de Ouro Preto, 258 CEP 96077-000
Pelotas - Rio Grande do Sul - Brasil
E-mail: juweykamp@hotmail.com 